\title{
On Air Pollution Monitoring Systems using IoT
}

\author{
K. Ranjith Reddy
}

\begin{abstract}
In the present day scenario, pollution caused by humans affects all the parameters of the environment (air, water, soil and sound). Air pollution is becoming a major concern in urban areas can expose the people to health issues like asthma attacks, lung problems, heart diseases etc. In this paper, two different methods are studied in monitoring the air quality of an environment. The first method involves usage of AWS cloud with a microcontroller and sensor. The second method uses Arduino along with Wi- Fi module and a sensor for measuring the level of pollutants and monitoring the air quality of a given environment.
\end{abstract}

Keywords-Air pollution, AWS cloud, Sensors, Arduino.

\section{INTRODUCTION}

Air pollution is one of the major challenge in environmental and health safety in world today. It is caused by industrialization and increase in the vehicles which in turn is increasing the harmful gases in atmosphere. Air pollution can cause health problems related to breathing, allergies and harmful diseases. According to WHO reports, 9 out of 10 people breathe pollutants in the air and an estimated 7 million people die every year.Across the world, urban and rural areas are seeing toxic pollutants in the air exceed the average annual values proposed by WHO's air quality guidelines. So, an air pollution monitoring system helps in getting the data related to the toxic level of gases present in the environment in PPM. This readings are stored and can be used for taking controlling measures of reducing the air pollution. The level of the toxic gases allowed and their measurement techniques are given by National Ambient Air Quality Standards.

\section{TABLE 1 .}

\section{NATIONAL AMBIENT AIR QUALITY STANDARDS (NAAQS)}

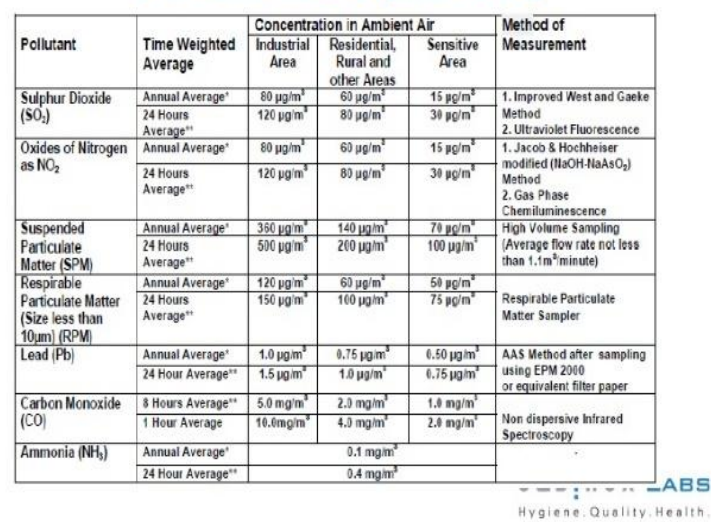

Revised Manuscript Received on 14 August, 2019. College for Women(Autonomous), Secunderabad-TS kranjithreddy1@gmail.com
K.Ranjith Reddy, Asst. Professor, Dept. of ECE, Malla Reddy Engineering

\section{LITERATURE SURVEY}

The measurement of air pollution can be basically done by fixed stations which give a wide range of pollutants. The fixed stations are not used more frequently as level of pollution varies according to the human activities and the environment in which it is placed. The cost involved in placement and maintenance of permanent station is also costly. Hence, low cost air pollution monitoring systems can be designed using IoT. A cloud based platform using IoT is used where smart phones are used in collection of data along with the sensors. It is useful in poor and developing countries where air quality is low. Another technique used for monitoring the air quality is by using a device such as Arduino or Raspberry Pi for conversion of sensor to digital value and to be connected to $\mathrm{Wi}-\mathrm{Fi}$ Module for transmissions Techniques

There are many different techniques used for collection of the pollutants present in the air based on the technique and the devices used.

\section{TECHNIQUES USED}

\section{Method 1 : Using AWS Cloud}

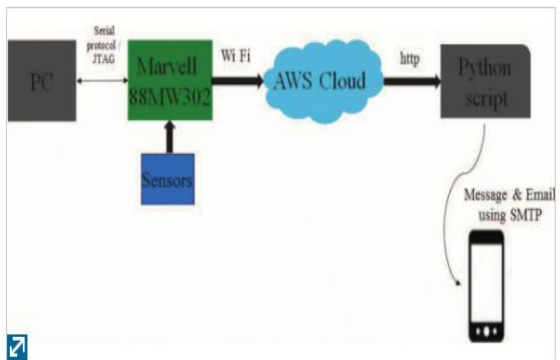

团

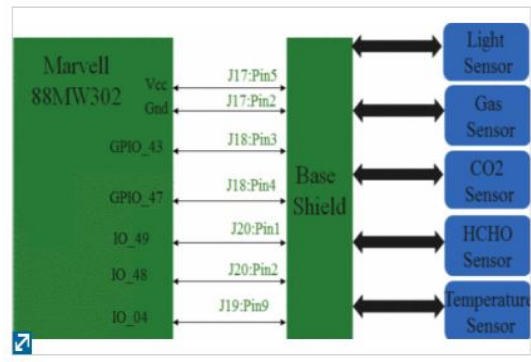

Fig 1 :Air quality monitoring system using AWS cloud

In this technique, sensor sends the measured data periodically to the server as well as to the AWS cloud. The low cost sensors are used to measure the data all over the city which can be accessed anywhere anytime anyone.

This system uses Maxwell 88MW302 for conversion of the sensor data into digital data which can be transmitted to the AWS Cloud. The maximum number of the sensors that can be connected to the base shield is 16 . 


\section{On Air Pollution Monitoring Systems using IoT}

The sensors which are used in the present system are Light sensor, Gas sensor, $\mathrm{CO} 2$ sensor, $\mathrm{HCHO}$ sensor and Temperature sensor. Maxwell 88MW302 board is Marvell EZ-Connect Wi-Fi microcontroller system-on-chip (SoC), with I/O interfaces including SPI, I2C, UART, 12S, PWM, ADC, DAC etc.AWS IoT provided by Amazon Web Services is a cloud platform that allows to connectdevices easily and provides secured interactions with other devices and Cloud services by using Wi- Fi. Python script is used to collect the AWS data on mobile phone.

\section{Method 2 : Using Arduino}

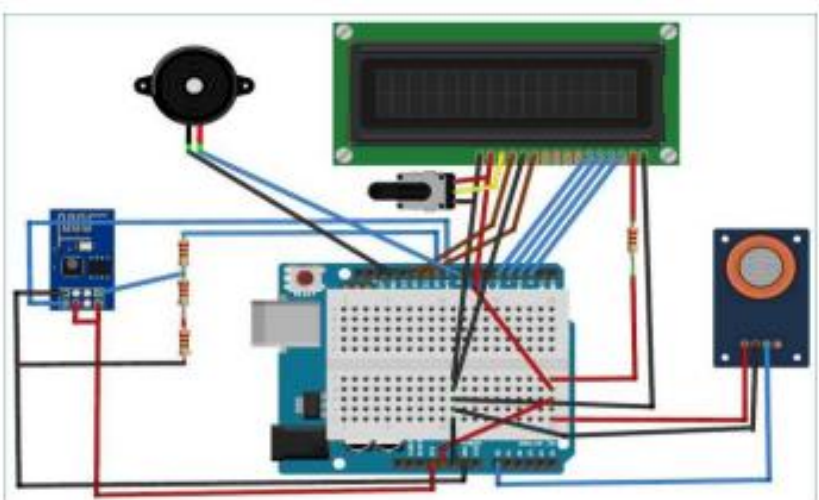

Fig 2 : Air quality monitoring system using Arduino

In this system, the components involved are Arduino, gas sensor, Wi- Fi module, LCD and resistors. The gas sensor used is MQ135 which is made of $\mathrm{SnO} 2$ and can detect toxic level upto 10,000 ppm with 5V AC/DC in CO2, sulphide, ammonia gas and benzene steam. The Wi- Fi module used is ESP8266 which transmits the data collected from sensor. The data measured from sensor goes to the Arduino and displayed on LCD as well as transmitted using the Wi- Fi module.

\section{RESULTS}

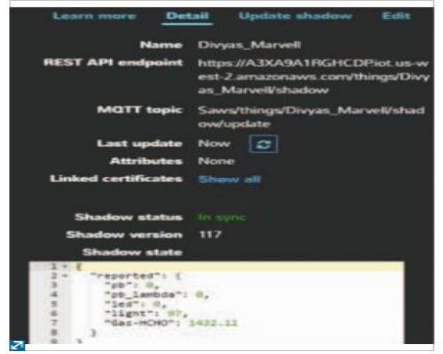

Fig 3 : Result of Air quality monitoring system using AWS Cloud

The output of the technique of measuring the air quality using AWS Cloud shows that the level of pollutants is less in morning and increasing rapidly as human activities are increasing.

The output of the technique using Arduino displays a message "Fresh Air" when sensor data is less than 1000 PPm , "Poor Air" when output $=1000$ PPm and "Harmful Air " when it is 2000PPm. The data statistics can also be shown on a webpage using Wi- Fi module.

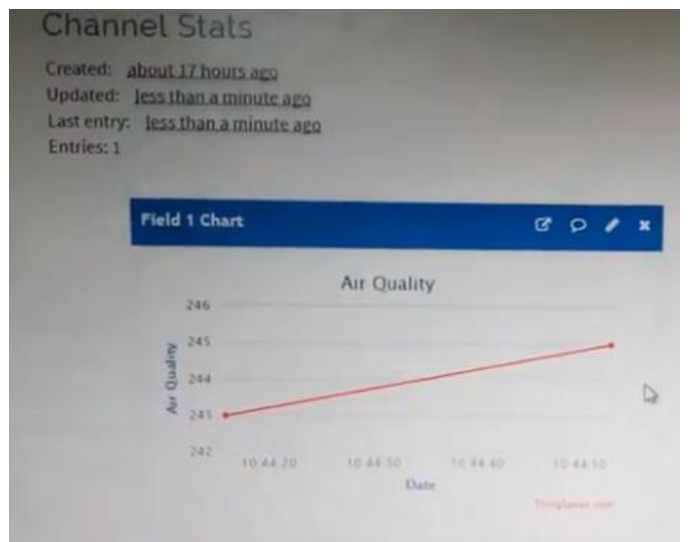

Fig 4 : Output of Air Quality Monitoring system using Arduino

\section{CONCLUSION}

The first method using AWS cloud is a low cost device which can be used to be implemented to measure the air quality for indoor environments. The second method where an Arduino is used for monitoring of the air quality can be well or both indoor and outdoor conditions. The quality of the toxic pollutants present in air from both the methods shows that the environment is less from mid night to morning, it increases in proportion to the human activities in the environment.

\section{REFERENCES}

1. https://ieeexplore.ieee.org/document/7857352/authors\#authors

2. https://www.irjet.net/archives/V4/i10/IRJET-V4I10207.pdf

3. https://www.ijser.org/researchpaper/IOT-Based-Air-Pollution-Monitoring -System.pdf

4. [4] Mohammad Jabirullah, J.Surendiran, Rajeev Arya," LOS IDENTIFICATION AND ITERATIVE LOCALIZATION OF WIRELESS SENSOR NETWORKS “, International Journal of Pure and Applied Mathematics Volume 119 No. 12 2018, 14367-14371 ISSN: 1314-3395.

5. [5] Telagam, N., Nanjundan, M., Kandasamy, N., \& Naidu, S. Cruise Control of Phase Irrigation Motor Using SparkFun Sensor. International Journal of Online Engineering (iJOE), 13(08), 192-198.

6. [6] Telagam, N., Kandasamy, N., \& Nanjundan, M. (2017). Smart Sensor Network Based High Quality Air Pollution Monitoring System Using Labview. International Journal of Online Engineering (iJOE), 13(08), 79-87.

7. networks," IEEE Trans. Inf. Theory, vol. 49, no. 10, pp. 2415-2425, Oct. 2003.

8. J. Laneman, D. Tse, and G. W. Wornell, "Cooperative diversity in wireless networks: Efficient protocols and outage behavior,” IEEE Trans. Inf. Theory, vol. 50, no. 12, pp. 3062-3080, Dec. 2004.

9. Y. Jing and B. Hassibi, "Distributed space-time coding in wireless relay networks," IEEE Trans. Wireless Commun., vol. 5, no. 12, pp 3524-3536, Dec. 2006.

10. S. Yiu, R. Schober, and L. Lampe, "Distributed space-time block coding," 